\title{
EFFECT OF DIODE LASER IRRADIATION ON INTERLEUKIN-8 PRODUCTION BY HUMAN NEUTROPHILS
}

\author{
Shinsaku Fukuda ${ }^{1}$, Ippei Takahashi², Takashi Umeda², Yuji Fujimaki², Takao Oyama², \\ Shigeyuki Nakaji ${ }^{2}$, Toshiaki Tsukamoto ${ }^{2}$ and Tadashi Shimoyama ${ }^{1}$ \\ 1: First Department of Internal Medicine, Hirosaki University School of Medicine and \\ 2: Department of Social Medicine, Hirosaki University School of Medicine
}

\begin{abstract}
Low reactive-level laser therapy (LLLT) is a safe and easy therapeutic modality and now utilized in the treatment of inflammatory conditions. However, the clinical evidence base for such application remains sparse. This study was designed to examine the effects of LLLT on production of interleukin-8 (IL-8) by human neutrophils. Neutrophils, separated from blood drawn from 10 healthy volunteers, received laser irradiation $(150 \mathrm{~mW} / \mathrm{cm} 2)$ for 30 or $60 \mathrm{sec}$ at $60 \mathrm{~min}$ prior to or just before the stimulation with opsonized zymosan. These conditions had different effects on production of reactive oxygen species by neutrophils. After the incubation at $37^{\circ} \mathrm{C}$ for 3 and 9 hours, the concentration of IL- 8 was measured by ELISA. The concentration of IL- 8 was higher when neutrophils were incubated longer. However, the levels of IL-8 production by irradiated neutrophils did not differ from those of unirradiated neutrophils even under the conditions whereby both the period of irradiation and the duration from irradiation to stimulation were different. IL-8 production by neutrophils may not play a role in the therapeutic effects of LLLT in inflammatory conditions.
\end{abstract}

Key words: laser irradiation, neutrophil, interleukin-8

\section{Introduction}

Chemokines play a very important role as far as white blood cell chemotaxis is concerned, especially chemotaxis to inflammatory lesions ${ }^{(1)}$. Chemokines are classified into four subfamilies from their structural characteristics. In particular, the CXC chemokine subfamily, members of which have a single amino acid between the first two cysteines of the characteristic four cysteine motif, has an very important role in neutrophil-mediated inflammation. One of the best-known CXC chemokines is interleukin-8 (IL-8), which is mobilized from the bone marrow and is activated against neutrophils through discharge of the lysosome enzymes, production of Roicotorien B4 and reactive oxygen species, and the induction of adhesion factors to neutrophils, and plays a very important role in acute and active inflammation. ${ }^{(2)}$

Activated neutrophils infiltrate inflamed tissue, and reactive oxygen species (ROS) are thought to be involved with damage to tissue in an

Addressee for Correspondence:

Shigeyuki Nakaji MD PhD,

Department of Social Medicine

Hirosaki University School of Medicine

5 Zaifu-cho, Hirosaki,

036-8562 Japan,

Tel. +81-172-395037, Fax. +81-172-395038,

E-mail.nakaji@cc.hirosaki-u.ac.jp inflammatory state. (3) It is known that LLLT irradiation is useful in the treatment of skin ulcers, ${ }^{(4)}$ oral apthae ${ }^{(5)}$ and arthritis. ${ }^{(6)}$ However, the exact mechanism in these entities remains unknown except for an increasing effect on blood flow. ${ }^{(7)}$ We have already reported one possible mechanism through which LLLT irradiation suppresses ROS production. ${ }^{(8)}$

On the other hand, up to the present, no report has examined the relationship between IL-8 and LLLT irradiation. A high rate of production of IL-8 is seen from neutrophils which have infiltrated inflamed tissue. Therefore, we hypothesized that, if LLLT irradiation could decrease neutrophil IL-8 production, the decreased levels of IL-8 would subsequently inhibit inflammation. In this study, we therefore examined how LLLT irradiation affected the IL-8 production of neutrophils.

\section{Materials and Methods}

Neutrophil suspension preparation and opsonization of zymosan

Normal pooled human neutrophils were obtained from the peripheral blood of 10 healthy adult male volunteers and separated by Histopaque density gradient centrifugation, according to the method already reported. ${ }^{(8)}$

Manuscript received: February 2005

Accepted for publication: March 2005 
Briefly, human blood was diluted with the same volume of HBSS containing heparin $(5 \mathrm{U} / \mathrm{ml})$ and this heparinized diluted blood was then settled onto Histopaque 1077 layered over Histopaque 1119 (Sigma, USA) and then centrifuged at $500 \mathrm{~g}$ at $4{ }^{\circ} \mathrm{C}$ for $30 \mathrm{~min}$. The neutrophil rich layer was collected and washed twice in HBSS and the neutrophils were resuspended to 2000 cells/ $\mu$ l using an automatic blood cell counter (Coulter MD II, Coulter Co. Ltd., Tokyo, Japan).

Opsonized zymosan (OZ) was used to stimulate neutrophil phagocytosis. Zymosan from Saccharomyces cerevisiae was employed for opsonized particles. Zymosan A (Sigma, USA) was suspended in Hank's balanced salt solution (HBSS) at a concentration of 5 $\mathrm{mg} / \mathrm{ml}$ and then opsonization was performed by adding the zymosan suspension to the serum samples to a final concentration of $13 \%$ and incubating at $37{ }^{\circ} \mathrm{C}$ for 30 $\min$.

Laser irradiation of neutrophils

A GaAlAs semiconductor laser, the Luketron MDL-1000 (Mochida Pharmaceutical Co., Tokyo, Japan), at a wavelength of $830 \mathrm{~nm}$ and an output power of $1000 \mathrm{~mW}$ in continuous wave was used for the laser irradiation. One of the features of this system is the fiberoptic delivery system which allows homogeneous irradiation of fairly large areas due to the multimode characteristics of the beam.
Wells were irradiated with the laser before and after incubation with an incident irradiance of 150 $\mathrm{mW} / \mathrm{cm}^{2}$. In a previous study we had ascertained that irradiation $60 \mathrm{~min}$ before stimulation of the neutrophils induced the greatest suppression of ROS production, ${ }^{(8)}$ so that protocol was followed in the current study. Irradiation times of 30 and $60 \mathrm{sec}$ were used, giving incident radiant fluences of 4.5 and $9 \mathrm{~J} / \mathrm{cm}^{2}$. These exposure times were chosen as being representative of popular clinical parameters. Following incubation, OZ was added to a final density of $1 \mathrm{mg} / \mathrm{ml}$, and incubation was continued at $37^{\circ} \mathrm{C}$. A control set of neutrophils was handled in exactly the same way as the irradiated sets including incubation, but did not receive any laser irradiation.

\section{Measurement of IL-8}

The samples in the control and experimental wells were removed after 3 or 9 hours, and centrifuged for ten minutes at $1000 \mathrm{~g}$. The IL-8 density in the supernatant was measured with an enzyme-linked immunosorbent assay (ELISA; BioSource Co., Camarillo, CA, USA).

\section{Statistical analysis}

All the measurement were given as mean values \pm standard deviation (SD). One-way ANOVA was done to test the differences in IL-8 density among three samples (immediately before irradiation, 60 minutes before irradiation and no irradiation) with post hoc test. Values of less

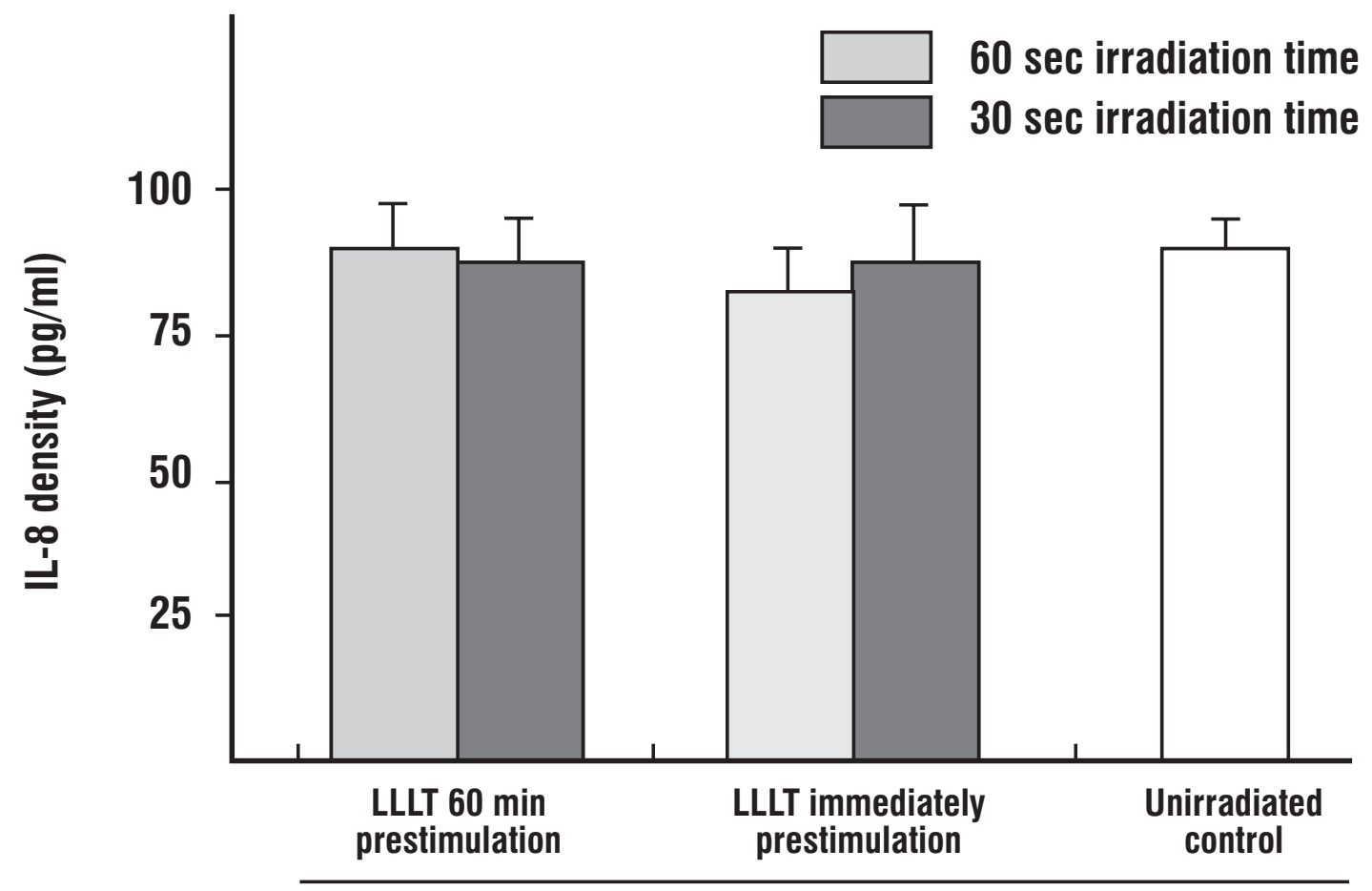

Cell groups

Fig 1: IL-8 concentration in irradiated and unirradiated neutrophil supernatant 3 hours after stimulation with opsonized zymosan 


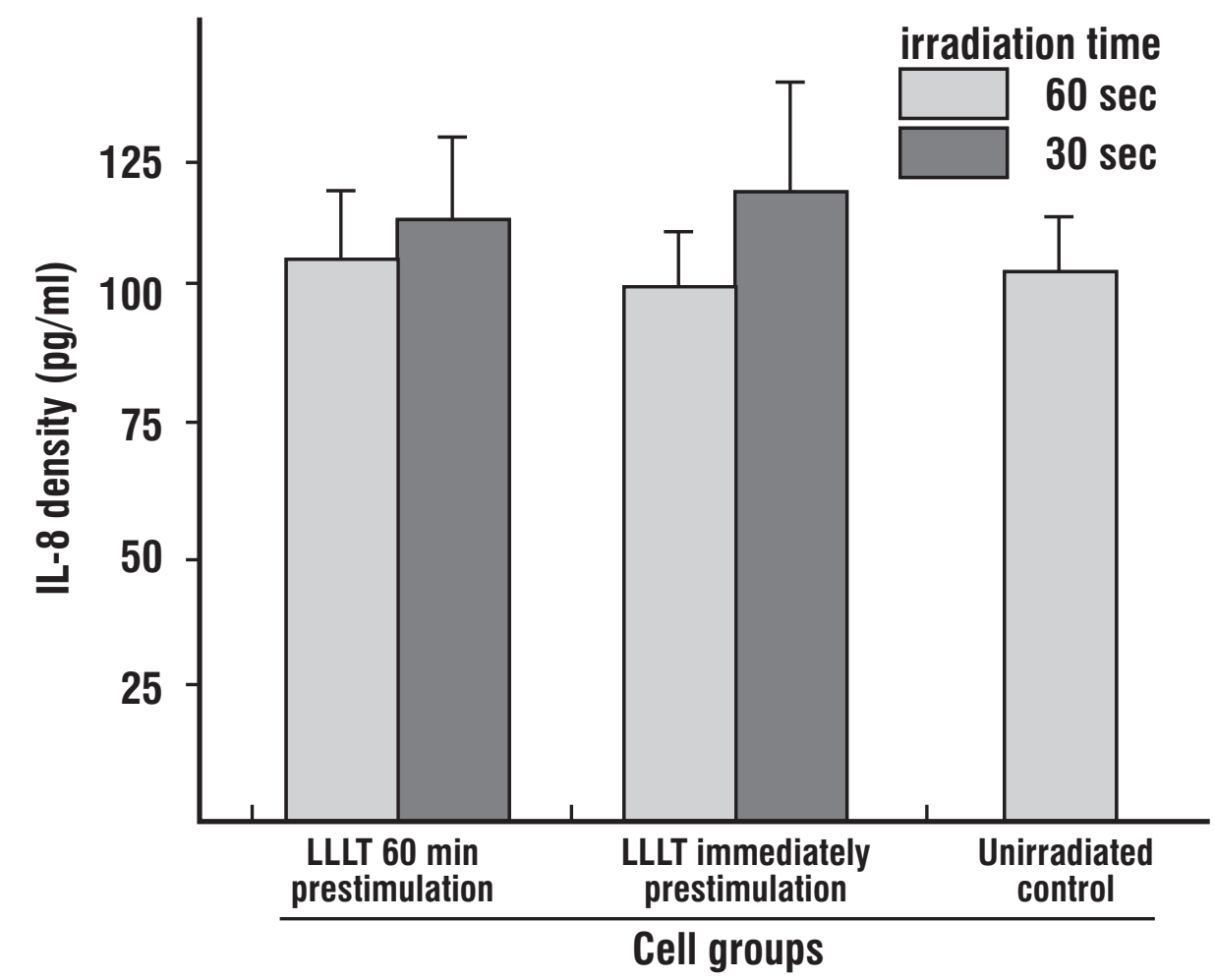

Fig 2: IL-8 concentration in irradiated and unirradiated neutrophil supernatant 9 hours after stimulation with opsonized zymosan

than P 5\% were evaluated as significant.

\section{Results}

The IL-8 density from the unirradiated control neutrophils was $89.1 \pm 16.7 \mathrm{pg} / \mathrm{ml}$ (Figure 1). When the neutrophils were irradiated with the laser immediately before stimulation, the IL- 8 density was $88.7 \pm 12.4$ $\mathrm{pg} / \mathrm{ml}$ following at 30-second exposure, and $81.0 \pm 10.8$ $\mathrm{pg} / \mathrm{ml}$ following a 60 -second exposure. Although there was a decreasing trend, there was no significant difference between either of the exposure times and the unirradiated control, or between the two irradiation times themselves.

When the laser was used to irradiate the neutrophils 60 minutes before neutrophil stimulation, the IL-8 density was $83.0 \pm 12.2 \mathrm{pg} / \mathrm{ml}$ following the 30 -second irradiation, and $88.2 \pm 15.9 \mathrm{pg} / \mathrm{ml}$ following the 60 -second irradiation. Once again there were no statistically significant differences between IL-8 density from the irradiated and unirradiated cells. In other words, at the parameters used in the present study IL- 8 production from neutrophils did not depend on either the timing or the duration of the laser irradiation.

Next, the IL-8 density after 9 hours of neutrophil stimulation is shown in Figure 2. The IL-8 density was $105.4 \pm 12.5 \mathrm{pg} / \mathrm{ml}$ in the unirradiated control neutrophils. When the neutrophils were irradiated with the la- ser immediately before stimulation of the neutrophils, the IL-8 density was $117.5 \pm 13.9 \mathrm{pg} / \mathrm{ml}$ following the 30 -second exposure, and $81.0 \pm 10.8 \mathrm{pg} / \mathrm{ml}$ for the 60 -second irradiation. No significant difference was seen in IL-8 density between the irradiated and unirradiated neutrophils, or between the two doses. When the neutrophils were irradiated 60 minutes before neutrophil stimulation, the IL-8 density was $114.1 \pm 17.4 \mathrm{pg} / \mathrm{ml}$ for the 30-second exposure time, and $108.5 \pm 18.8 \mathrm{pg} / \mathrm{ml}$ for the 60 -second irradiation. Therefore, IL-8 production from neutrophil did not depend either on the timing or duration of the laser irradiation, though it did depend on the incubation time.

\section{Discussion}

LLLT irradiation may be effective for the treatment of arthritis, as has been suggested in the literature. ${ }^{(9)}$ In arthritis, neutrophil infiltration into the tissue is seen. Furthermore, in previous reports of an experiment in the rat model, an injection of IL-8 into the inside of the joint capsule induced inflammation similar to chronic arthritis. ${ }^{(10)}$ Such evidence suggests that neutrophil infiltration may play some role in arthritis. Furthermore, it is known that IL-8 is associated with tissue damage via increased ROS production from neutrophils. ${ }^{(1)}$ Indeed, administering anti-IL-8 antibody reduces neutrophil infiltration into the joint capsule and joint swelling.(11) 
These findings mean that IL-8 induces neutrophil infiltration and tissue damage.

ROS from activated neutrophil by such as bacterial lipopolysaccharide should induce not only arthritis but also ulcers of the digestive tract or aphtae of the oral cavity. ${ }^{(12)}$ It is known that the level of the neutrophil infiltration into tissue is associated with the amount of local IL-8, and it has been suggested that the existence of IL-8 is associated with ulcer formation. ${ }^{(13,14)}$ Moreover, it has been shown in a recent report that gingival fibroblastoma cell production of IL- $1 \beta$ is inhibited by irradiation with the GaAlAs laser. This suggests a possible role for LLLT radiation through an anti- inflammatory action via inhibition of cytokine production. ${ }^{(15)}$

In our previous studies, LLLT inhibited the ROS production from neutrophils, suggesting a possible application pain relief or wound healing. ${ }^{(8)}$ In the current study, when neutrophils were irradiated with LLLT at same level which significantly inhibited the ROS production in our earlier study, IL-8 production showed no change. Therefore, LLLT irradiation did not have any effect on IL-8 production when the neutrophils was stimulated with zymosan (via the CD11b, CD16 receptors $\left.{ }^{(16)}\right)$. However, if a stimulating factor other than zymosan were to be used, IL- 8 production might be changed following LLLT irradiation. Furthermore, since there are complex cytokine networks in vivo, further study is required in the future.

\section{Acknowledgements}

We would like to express our sincere thanks to Dr. Kazuo Sugawara of the Department of Hygiene, Hirosaki University School of Medicine for his suggestions during the preparation of this manuscript.

\section{References}

1: Luster AD (1998): Mechanisms of disease: chemokines-chemotactic cytokines that mediate inflammation. N EnglJ Med, 338: 436-445.

2: Yosie O, Nomiyama H, Miyasaka M (eds): Chemokine handbook, Tokyo, Shunjun-sha Co. Ltd., 2000

3: Rosenfeld ME. Inflammation, lipids, and free radicals: lessons learned from the atherogenic process. Semin Reprod
Endocrinol, 16: 249-61.

4: Basford JR. The clinical and experimental status of low energy laser therapy (1989): Crit Rev Phys Rehab Med, 1: 1-9.

5: Pinheiro AL, Cavalcanti ET, Pinheiro TI, et al (1998): Low-level laser therapy is an important tool to treat disorders of the maxillofacial region. J Clin Laser Med Surg, 16: 223-6.

6: Amano A, Miyagi K, Azuma T, et al. Histological studies on the rheumatoid synovial membrane irradiated with a low energy laser. Laser Surg Med, 15: 290-4.

7: Kami T, Yoshimura Y, Nakajima T, Ohshiro T, Fujino T (1985): Effects of low-power diode lasers on flap survival. Ann Plast Surg, 14: 278-83.

8: Fujimaki Y, Shimoyama T, Liu Q, Umeda T, Nakaji S, Sugawara K (2003): Low-level laser irradiation attenuates production of reactive oxygen species by human neutrophils. J Clin Laser Med Surg, 21: 165-170.

9 Asada K, Yutani Y, Sakawa A, and Shimazu A (1991): Clinical application of GaAlAs $830 \mathrm{~nm}$ diode laser in treatment of rheumatoid arthritis. Laser Therapy, 3: 77-82.

10: Akahoshi T, Endo H, Kondo H, et al (1994): Essential involvement of interleukin- 8 in neutrophil recruitment in rabbits with acute experimental arthritis induced by lipopolysaccharide and interleukin-1. Lymphokine Cytokine Res, 13: 113-116.

11: Hachicha M, Naccache PH, McColl SR (1995): Inflammatory microcrystals differentially regulate the secretion of macrophage inflammatory protein 1 and interleukin 8 by human neutrophils: a possible mechanism of neutrophil recruitment to sites of inflammation in synovitis.J Exp Med, 182: 2019-2025.

12: Chapple IL (1997): Reactive oxygen species and antioxidants in inflammatory diseases. J Clin Periodontol, 24: 287-96.

13: T Shimoyama, SM Everett, MF Dixon, ATR Axon, JE Crabtree (1998): Chemokine mRNA expression in gastric mucosa is associated with Helicobacter pylori cagA positivity and severity of gastritis. J Clin Pathol, 51: 765-770.

14: Mazzucchelli L, Hauser C, Zgraggen K, et al (1994): Expression of interleukin- 8 gene in inflammatory bowel disease is related to the histological grade of active inflammation. Am J Pathol, 144: 997-1007,

15: Nomura K, Yamaguchi M, Abiko Y(2001): Inhibition of interleukin-1beta production and gene expression in human gingival fibroblasts by low-energy laser irradiation. Lasers Med Sci, 16: 218-223.

16: Rossi $\mathrm{F}(1986)$ : The $\mathrm{O}_{2}$-forming NADPH-oxidase of phagocytes nature, mechanisms of activation and function. Biochemi Biophys Acta, 853: 65-89. 